\title{
Crisis de gobernanza y gobernabilidad y sus implicancias en el uso inadecuado del agua subterránea, caso acuífero costero de La Yarada, Tacna, Perú
}

\author{
Governability and governance crisis its implications in the inadequate use of \\ groundwater, case coastal aquifer of La Yarada, Tacna, Perú
}

\author{
Edwin Pino V. ${ }^{*}$, Eduardo Chávarri V.2, Lia Ramos F. ${ }^{2}$
}

\begin{abstract}
RESUMEN
La crisis de gobernabilidad y gobernanza en el uso del agua subterránea en el acuífero costero de La Yarada es un elemento que contribuye al agotamiento y deterioro de la calidad del agua subterránea, por procesos de intrusión marina. Este trabajo busca caracterizar la crisis de gobernabilidad en el uso del agua subterránea, bajo las condiciones actuales de explotación, y establecer sus implicancias en el área de estudio, interpretando la normatividad nacional e internacional vigente y aplicable a este tipo de sistema. Este problema, en todos sus términos, altera y rebasa las leyes respecto a los regímenes de la gestión y limitaciones en disponibilidad de los recursos hídricos subterráneos. De no tomarse acciones inmediatas por parte del aparato gubernamental, la crisis del sistema podría volverse irreversible, con el consiguiente perjuicio económico que esto acarrea. En la zona se cuenta con grandes extensiones de plantaciones de olivo, que son el soporte de la actividad económica en la región. En tal sentido, resulta necesario contar con un marco normativo específico para las aguas subterráneas que reconozca su particularidad. Se debe consolidar y hacer fuerte la institucionalidad en materia de Derecho de Aguas, y esta no es una necesidad única para el caso de las aguas subterráneas, sino de manera general para los recursos hídricos en todas sus formas en nuestro país.
\end{abstract}

Palabras clave : crisis de gobernanza, gobernabilidad, uso inadecuado del agua subterránea, acuífero La Yarada

\begin{abstract}
The governability and governance crisis in the use of groundwater in the coastal aquifer of La Yarada, is an element that contributes to the depletion and deterioration of the quality of groundwater, by processes of marine intrusion. This paper is to characterize the governance crisis in use of the groundwater, under the current conditions of exploitation and establish its implications in the study area, interpreting the current national and international regulations applicable to this type of system. This problem, in all its terms, alters and exceeds the laws regarding the management regimes and limitations in the availability of underground water resources, if immediate action is not taken by the governmental apparatus, the crisis of the system could become irreversible with the consequent economic damage that this entails. In the area there are large areas of olive plantations, which are the support of economic activity in the region. In this sense, it is necessary to have a specific regulatory framework for groundwater that recognizes its particularity, it must consolidate and make strong the institutionality in the area of water law, not being a unique need to the case of groundwater, but in a general to water resources in all its forms in our country.
\end{abstract}

Key words : governability crisis, governance, inadequate use of groundwater, La Yarada aquifer

\section{Introducción y revisión bibliográfica}

La región de Tacna tiene como limitante principal para su desarrollo la severa escasez de agua, razón por la cual los agricultores establecidos en las pampas de La Yarada usan agua subterránea, siendo esta la única fuente de abastecimiento. En la actualidad, el acuífero está sobreexplotado, lo que trae como consecuencia un gradual y permanente descenso del nivel freático, comprometiendo sus reservas no renovables y causando el fenómeno de intrusión marina. Ante esta problemática, la Autoridad Nacional del Agua (ANA) ha elaborado un "Plan de ordenamiento de la explotación del agua subterránea en el acuífero Caplina", con el propósito de explotar el recurso hídrico de manera eficiente, organizada y

\footnotetext{
Universidad Nacional Jorge Basadre Grohmann, Tacna, Perú.

Universidad Nacional Agraria La Molina, Lima, Perú.

Autor por correspondencia: epino68@hotmail.com, epinov@unjbg.edu.pe
}

Fecha de Recepción: 31 mayo, 2018.

Fecha de Aceptación: 27 julio, 2018. 
sostenida. Asimismo, se evaluaron las condiciones y características hidrogeológicas del subsuelo del acuífero, lo que permitió determinar la reserva explotable de agua en cantidad y calidad, factible de explotar, en forma sostenible a largo plazo, y proponer medidas para mejorar su gestión y conservación. Dicho plan no fue implementado en ninguno de sus términos por problemas graves de gobernabilidad.

El acuífero costero de La Yarada se encuentra ubicado en la región de Tacna al sur de Perú, límite con Chile y Bolivia (Figura 1). Esta región, caracterizada por la escasez de agua, está situada en la cabecera del desierto de Atacama (Chile), y esa condición se ha agudizado en las últimas décadas debido a la frecuente presencia de sequías, la ampliación de la frontera agrícola no autorizada con la consecuente incorporación de nuevos pozos sin licencia de explotación de aguas subterráneas, el acelerado crecimiento poblacional de la ciudad de Tacna y las múltiples actividades económicas que esto trae consigo. Sin embargo, debido a la falta de un programa de explotación adecuado, el acuífero está siendo sobreexplotado, lo que ha traído como consecuencia un gradual y permanente descenso del nivel freático, comprometiendo sus reservas no renovables. Esto ha causado el fenómeno de la intrusión marina, debido a la alta concentración de pozos de explotación de aguas subterráneas en la zona próxima a la línea del mar. La rápida expansión económica genera serios problemas para el uso del agua subterránea en zonas áridas, las que normalmente tienen altas tasas de agotamiento (Mohamed, et al., 2017).

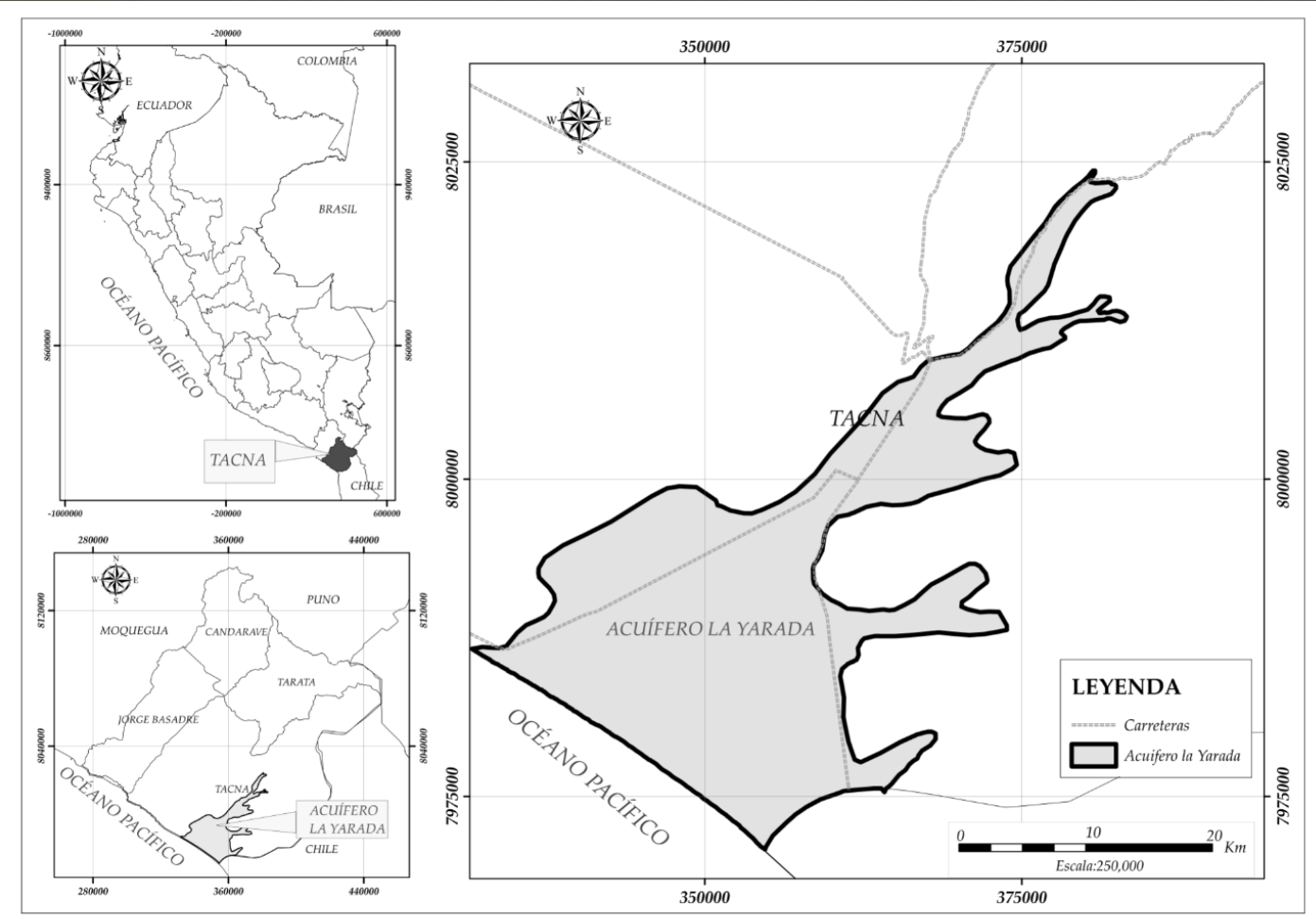

Figura 1. Ubicación de la zona de estudio

Una situación similar a la del acuífero de $\mathrm{La}$ Yarada se presenta en el estado de California, donde el agua subterránea es una fuente vital que proporciona suministros para las áreas urbanas y rurales. Se usa en una gran economía agrícola, beneficia a los peces, hábitats y ecosistemas, y cubre casi el 40 por ciento de la demanda de agua del estado en un año promedio. Durantelassequías, cuandolos suministros delasaguas superficiales son limitados, las aguas subterráneas ofrecen un amortiguador crítico, proporcionando un 
porcentaje más alto del suministro de agua (Borchers et al., 2015). En el año 2014 se previó que el uso de agua subterránea en todo el estado estaría cerca del 65 por ciento de la demanda total, debido a la naturaleza crítica de la sequía de ese periodo, por ausencia de precipitaciones. Los principales acuíferos del mundo, de los que dependen cientos de millones de personas, se agotan a un ritmo alarmante, según datos obtenidos a través de los satélites Grace y hechos públicos por la NASA. De los 37 acuíferos más grandes repartidos por todo el mundo, desde la India y China hasta EE UU o Francia, 21 han superado su punto de sostenibilidad, lo que significa que de ellos se ha extraído ya más agua de la que se ha incorporado a lo largo de los diez años de observación (Todd, 2015). El estudio de la NASA confirma las sospechas que ya tenían numerosos investigadores, especialmente en los casos de acuíferos que no son recargables por la lluvia. Asimismo, una preocupación en el ámbito de las ciencias ambientales y del mismo derecho ambiental es el notable deterioro y disminución de los humedales y de las aguas subterráneas, teniendo en cuenta que una de sus causas es el fenómeno del cambio climático (Minaverry, 2017).

A la par de los procesos naturales que generan problemas de déficit hídrico en los sistemas acuíferos, también se tienen dificultades de orden social. La gobernabilidad se da en el sentido de que se considera al Estado como el agente central de la conducción de la sociedad. Así, su preocupación se centra en "la capacidad de gobernar", considerando a la sociedad como la entidad a ser gobernada y administrada (Villalobos, 2015). Desde la perspectiva de la gobernabilidad, el problema y su solución emana de las capacidades del gobierno, con cierta independencia de la sociedad, mientras que la gobernanza parte del reconocimiento de que el gobierno carece de las capacidades suficientes para la resolución de los problemas sociales.

Por tanto, nos referiremos a la gobernabilidad cuando se trate de la parte institucional conferida al gobierno y sus instituciones, es decir, su capacidad y rango de acción; y utilizaremos gobernanza cuando nos refiramos a la acción conjunta del gobierno y la sociedad para un objetivo común (como el desarrollo), a fin de alcanzar un equilibrio. Para tomar decisiones adecuadas, con el fin de lograr metas de gestión integrada del agua, es necesario armonizar los intereses y la dinámica de las poblaciones con las condiciones y la dinámica propia del entorno donde ellas habitan, en particular con relación a las cuencas hidrográficas y el ciclo hidrológico (Dourojeanni y Jouravlev, 2001).

Se evidencia que la gobernabilidad tiene dos caras íntimamente relacionadas: la capacidad de formular políticas adecuadas y la capacidad de implementarlas. Esas capacidades requieren la construcción de consensos, el montaje de sistemas de gestión, leyes, agencias públicas nacionales y regionales, conocimientos e información, prácticas, y la administración adecuada del sistema que supone participación social y desarrollo de competencias (Rodríguez, 2005). En el caso específico de Chile, se dispone de un sistema de gestión del agua (SGA-CH) que puede ser catalogado como único en su tipo, y que considera el agua como una mercancía transable. Este sistema se sustenta legalmente en el Código Nacional del Agua (CNA), el cual se encuentra en el mismo nivel jerárquico que el Código Civil (Retamal et al., 2013).

Luego de una exhaustiva revisión bibliográfica, se identifican siete versiones de gobernabilidad que resultan muy pertinentes en razón de su grado de expresión en el contexto colombiano y que son aplicables al ámbito sudamericano. Estas interpretaciones se muestran en las Figuras 2 y 3 (Zamudio, 2012).

Si queremos lograr un óptimo aprovechamiento de nuestros valiosos recursos en aguas subterráneas y garantizar su sostenibilidad, debemos gestionarlos y manejarlos con cuidado (UNESCO, 2015). En la práctica se da continuidad al esquema de gestión de carácter jerárquico y centralizado vigente desde hace más de cuatro décadas, y si bien la implementación de un modelo de gobernanza del agua desde la perspectiva de la Gestión Integrada de los Recursos Hídricos (GIRH) parece bastante lejana, existe la posibilidad de poner en marcha acciones públicas desde los territorios, a partir de las cuales se estructuren redes, articulen intereses y emprendan acciones colectivas (González, 2017).

La práctica ha demostrado que la gestión y protección de las aguas subterráneas suele ser muy difícil. Las aguas subterráneas interactúan con diversos componentes adicionales del entorno natural y a su vez se ven afectadas por estos (UNESCO, 2015). Los acuíferos transfronterizos también representan una oportunidad de integración y colaboración regional que incluye la anticipación, prevención de conflictos y competencia entre los usuarios del agua.

En la declaración de Dublín, según los encuentros internacionales sobre la gestión de recursos hídricos, 


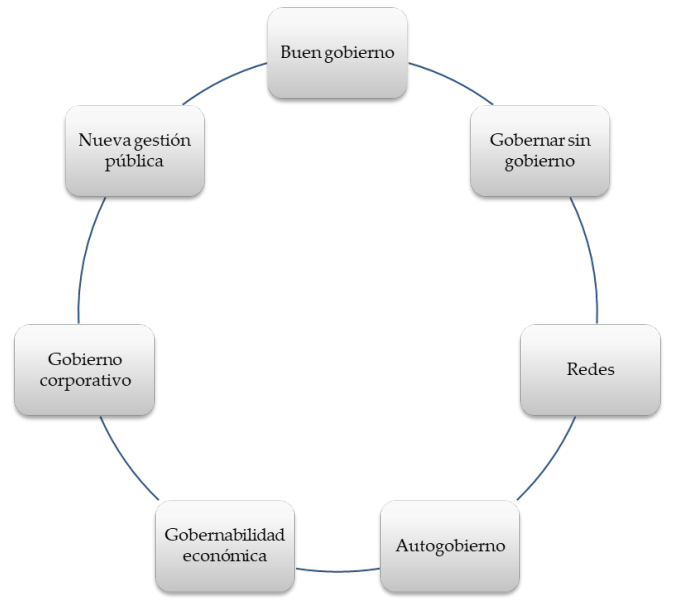

Figura 2. Enfoques de gobernabilidad

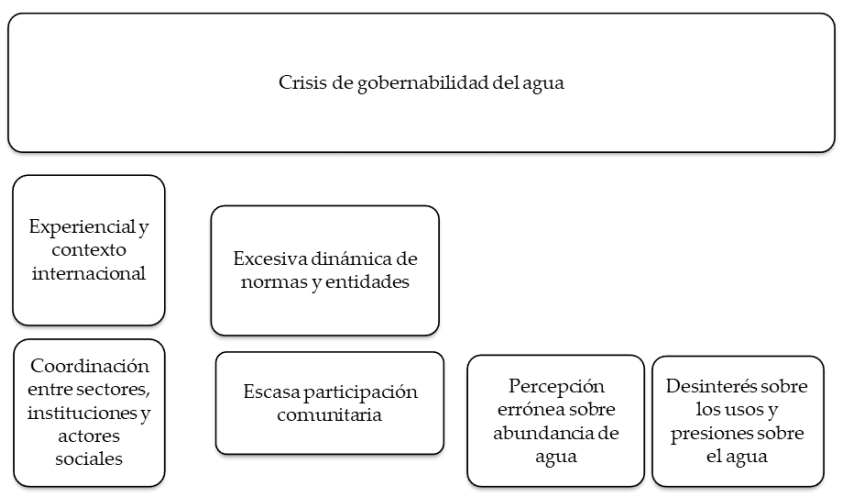

Figura 3. Algunos elementos sobre la crisis de gobernabilidad del agua

se reconoció que el agua es esencialmente un bien económico. Los economistas han insistido en la necesidad de reconocer que el agua es un bien económico, y entonces debería ser posible gobernar su distribución por medio del mercado (Terence y Jouravlev, 1998). Existe una gran cantidad de enfoques que explican la dificultad para mejorar los sistemas de gestión del agua, y entre ellos tenemos la falta de métodos para diseñar estrategias que permitan transitar paso a paso de una situación existente a una situación deseada (Dourojeanni y Jouravlev, 2001).

Los principios de Dublín y los planes de acción coherentes con ellos fueron recogidos en la Cumbre de la Tierra celebrada en Río de Janeiro, en el Capítulo 18 de la Agenda 21 (WWAP, 2006), en los siguientes términos: "Una ordenación global del agua dulce en cuanto recurso limitado y vulnerable y la integración de planes y programas hídricos sectoriales, dentro del plano de la política económica y social nacional, son medidas que revisten la máxima importancia entre las que se adopten en el decenio de 1990". Hay controversias sobre el tema de los resultados de los cambios en la gobernanza del agua. Esto, creemos, se puede caracterizar mejor como un problema de identificación de los efectos y se refiere a la cuestión de los efectos de los cambios en la gobernanza en una amplia variedad de fenómenos empíricos y asuntos normativos asociados (Kersbergen y Waarden, 2004).

La crisis de gobernanza y gobernabilidad en el uso del agua subterránea, caso acuífero costero de La Yarada, contribuye al deterioro de la calidad del agua subterránea, por procesos de contaminación por intrusión marina. En tal sentido, este trabajo busca caracterizar la crisis de gobernabilidad en el uso del 
agua subterránea, bajo las actuales condiciones de explotación, y establecer sus implicancias en el área de estudio perteneciente al acuífero de La Yarada, interpretando la normatividad nacional e internacional vigente aplicable a este tipo de uso.

\section{Materiales y métodos}

Según las características del caso estudiado, metodológicamente establecimos interrelaciones, donde se consultan estudios académicos y material oficial de dominio público. Bajo esta metodología, buscamos explicar la crisis de gobernanza y gobernabilidad en el uso del agua, en función de la legislación en agua subterránea, gestión integrada del agua, acción de la Autoridad del Agua y Organizaciones de Usuarios.

Existe documentación cronológicamente ordenada sobre el acuífero de La Yarada y en general sobre el agua subterránea en el país. Como técnica de análisis se utilizó el método de la hermenéutica jurídica: se contrastó la normativa de dos jurisdicciones del Perú, nacional y de la región de Tacna, específicamente en el caso del agua subterránea, y se analizaron instrumentos internacionales y selección de casos jurisprudenciales.

El trabajo de campo se organizó en función de la importancia de las instituciones ligadas a la problemática del uso del agua en el acuífero de La Yarada. El ente rector es la Autoridad Nacional del Agua, sin exceptuar directivas o decretos generados por el gobierno local y regional.

\section{Instrumentos internacionales sobre uso del agua subterránea}

Se puede afirmar que pocas veces el Derecho internacional ha tenido en cuenta el agua subterránea, y se sabe que existen muchos tratados del agua de superficie. El agua subterránea no está nominalmente incluida en el alcance de estos instrumentos, principalmente, si se la "relaciona" con las aguas territoriales de superficie. Algunos instrumentos legales contienen condiciones específicas del agua subterránea, y no la abordan con exclusividad. La FAO y la UNESCO aportan una variedad de instrumentos obligatorios y no obligatorios de Derecho internacional en este importante campo (Burchi y Mechlem, 2007).

Asimismo, existen directivas sobre recursos hídricos y tratados ambientales que contienen disposiciones sobre aguas subterráneas, como es el caso de la Convención de las Naciones Unidas de
Lucha contra la Desertificación en aquellos países que sufren sequía grave y/o desertificación, en particular en África. También se cuenta con la Convención de las Naciones Unidas sobre el derecho de los usos de los cursos de agua internacionales para fines distintos de la navegación. Estas disposiciones son importantes pero se generaliza para los recursos de toda índole y en el segundo caso para los cursos de agua internacionales, y no es específico para el tema del agua subterránea, siendo base estratégica para abordarlo.

Existen acuerdos interestatales sobre aguas subterráneas o disposiciones sobre aguas subterráneas. United States, Idaho-Washington, en forma específica el Acuerdo Interinstitucional en Materia de la Gestión Coordinada del Acuífero de Aguas Subterráneas Pullman-Moscow (Burchi y Mechlem, 2007), establece que la administración de los recursos hídricos subterráneos del acuífero estará de acuerdo con el plan de gestión de aguas subterráneas adoptado del Pullman Moscow Water Resources Committee (PMWRC), en la medida en que pueda implementarse y administrarse según las leyes de cada estado.

\section{Instrumentos nacionales sobre uso del agua subterránea}

En el Perú, el otorgamiento de derechos de uso de agua subterránea se encuentra regulado en el Título IV de la Ley de Recursos Hídricos (artículo 110). En la referida ley se precisan dos disposiciones adicionales: la primera señala que en caso de cese del aprovechamiento, bien sea permanente o temporal, los titulares de los derechos deberán adoptar las medidas de seguridad necesarias a fin de evitar ocasionar daños al acuífero y a terceros. La segunda señala que es obligación de los usuarios de aguas subterráneas instalar y mantener piezómetros en la cantidad y separación determinadas conforme lo disponga la autoridad correspondiente, información la cual deberá ser comunicada a la ANA (Cairampoma y Villegas, 2016).

Según Decreto Supremo D.S. N 080-84-AG del 6 de septiembre de 1984, se reservaron por dos años las aguas subterráneas de las pampas de La Yarada, y el D.S. $\mathrm{N}^{\circ}$ 020-87-AG del 1 de mayo de 1987 da una prórroga por dos años más. Mediante Resolución Ministerial N $0555-89-$ AG/DGAS del 5 de diciembre de 1989 se prohíbe la ejecución de obras destinadas a extraer aguas subterráneas en las pampas de La Yarada. Posteriormente, mediante 
R.M. N 696-98-AG del 16 de diciembre de 1998 y con base en el estudio hidrogeológico de las pampas de La Yarada, se declara la veda sobre el incremento de explotación de agua subterránea en el acuífero. El Tribunal Constitucional, mediante sentencia emitida en el expediente $\mathrm{N}^{\circ} 1290-2002-\mathrm{AC} / \mathrm{TC}$ se pronunció sobre la declaración de veda y la inaplicación de normas de regularización de licencias de agua en la zona declarada en veda.

La Ordenanza Regional N009-2004-CR/ GOB.REG.TACNA declaró de interés regional la intangibilidad y conservación de las aguas subterráneas y tierras eriazas del estado de las pampas de La Yarada. En el año 2006, mediante D.S. $\mathrm{N}^{\circ} 065$ 2006-AG, se declara de necesidad pública y de interés nacional la conservación y preservación del recurso hídrico del valle Caplina, ampliándose la veda a todo el acuífero Caplina (Art. 2). Esto es ratificado en el año 2009 mediante R.J. $\mathrm{N}^{\circ}$ 327-2009-ANA del 15 de junio, que declara zona de veda el acuífero del valle del río Caplina, donde está incluido el acuífero de La Yarada; la prohibición de ejecución de obras de explotación de aguas subterráneas y la disposición de que los Administradores Locales de Agua son los responsables del control y vigilancia de los acuíferos que se encuentran en sus respectivos ámbitos jurisdiccionales.

La R.J. N ${ }^{\circ}$ 201-2010-ANA, del 22 de marzo del 2010, ratifica las medidas para la conservación y preservación del agua subterránea, basada en el estudio "Modelamiento Numérico del Acuífero La Yarada", el cual concluye que existe una sobreexplotación del acuífero y recomienda mantener la veda de la explotación del sistema. Finalmente, en el año 2015, se emite el D.S. $\mathrm{N}^{\circ}$ 007-2015-MINAGRI, el cual en su segunda disposición complementaria señala: "Las zonas declaradas en veda mantienen su condición, procediéndose de manera excepcional y por única vez a formalizar o regularizar las licencias de uso de agua, conforme a lo establecido en el presente Decreto Supremo".

\section{La crisis de gobernanza y gobernabilidad}

La gobernabilidad se refiere a la gestión de asuntos colectivos e involucra la articulación de reglas de conducta y el acuerdo de principios para la asignación de recursos en el marco de una comunidad política (Healey, 1997). Asimismo, la gobernabilidad tiene que ver con la elección de los fines y valores que deben orientar a la sociedad (Green y Peterson,
2005). En el Perú podemos afirmar que no existe dispersión de disposiciones legales, ni profusión de leyes. Lo que sí estimamos encontrar son vacíos tanto conceptuales como de estrategia y acción, que resultan realmente importantes. En la mayoría de los casos, las disposiciones están desactualizadas y desvinculadas de la realidad regional y nacional. Asimismo, en la actualidad tenemos instrumentos importantes como el principio integrador en la gestión del agua, el cual está ausente en las acciones gubernamentales adoptadas para enfrentar la problemática del acuífero de La Yarada.

La fragmentación de las acciones respecto a la gestión del agua en el sistema acuífero es un problema relevante. Las instituciones actúan en forma aislada y sin coordinar acciones conjuntas necesarias para tratar el problema. Esto denota que se tiene un ente rector de las políticas de gestión del agua en el país, como es la Autoridad Nacional del Agua (ANA), que no cuenta con la solvencia técnica y normativa para ejecutar la legislación vigente en materia de guas sobre el acuífero de La Yarada. El gobierno regional y local no tiene participación en la gestión y territorialidad del agua en el sistema acuífero.

Existen limitaciones para la efectividad de la gobernabilidad y radican en la relación del agua subterránea con la sociedad. Se tiene la concepción del agua como un recurso inagotable, cultura de no pago del agua y falta de responsabilidad de protección ambiental. Respecto a las políticas públicas y sus estrategias de incorporación, estas son prácticamente nulas. Es notoria la fragmentación de políticas y, lo más importante, existe fragilidad de las instituciones del Estado relacionadas con el tema del agua subterránea, para el control del uso y su aprovechamiento. Asimismo es evidente la falta de planificación gubernamental para lograr un adecuado uso del agua. También podemos identificar una falta de educación sobre la preservación y uso sostenible del agua subterránea del sistema acuífero La Yarada.

La gestión del sistema es un problema latente. No se tiene clara la legislación sobre penalizaciones por delitos de uso de agua sin autorización o sin licencias o permisos otorgados. No se cuenta con un inventario confiable de los pozos existentes con autorización de uso y sin esta, por lo tanto no se puede precisar un balance hídrico por regiones y considerando niveles de vulnerabilidad. La incorporación de municipalidades, gobernaciones y otras instituciones en la gestión del agua subterránea, se busca a través de la conformación del Consejo de Recursos Hídricos de Cuenca Caplina 
- Locumba, creado por Decreto Supremo N ${ }^{\circ} 019$ 2013-AG. Este es un espacio de concertación en el cual las instituciones y organizaciones de la región, vinculadas a la gestión integrada de los recursos hídricos, pueden presentar sus necesidades, proyectos y reclamos, con la finalidad de planificar y coordinar el aprovechamiento sostenible del agua en la cuenca. En la conformación de este consejo se han reportado problemas graves. Algunos de sus miembros, en especial quien preside dicho consejo, no cumplen los requisitos señalados en la Ley $\mathrm{N}^{\circ} 29338$, Ley de Recursos Hídricos y su reglamento. Esta situación termina por establecer un clima de desconfianza respecto al incumplimiento de la ley, partiendo de la misma entidad rectora del uso y conservación del agua en el país, es decir, la ANA.

\section{Modelos de gobernabilidad y gobernanza}

Mejorar la gobernabilidad es la clave para hacer frente a la inseguridad del agua en los países en vías de desarrollo. La literatura no presta atención al estudio de las estructuras de incentivos, a la interdisciplinariedad y a la orientación con implicaciones políticas claras (Silva, 2016). En el modelo de Fung y Wright, el enfoque de gobernanza se presenta como una forma no jerárquica de gobierno, caracterizada por la cooperación con actores no estatales al interior de redes de decisión mixtas entre lo público y lo privado (Fung y Wright, 2003).

El modelo de gobernanza del agua de laUNESCO es un modelo teórico donde se enfocan temas gubernamentales, cuya finalidad es la formulación de políticas y su ejecución por parte del Estado. El modelo se integra por las dimensiones social, económica, de la capacitación política y de la sostenibilidad medioambiental (UNESCO, 2006). En el modelo leviatán hidráulico de Musetta se hace énfasis en un Estado centralizado, fuerte en términos de presencia estatal, un Estado que planifica y desarrolla, que construye grandes obras de infraestructura hidráulica (diques, represas, sistemas de riego) y en esto se mide el potencial de su fuerza. Es a su vez un Estado que organiza a los demás actores de la economía y de la sociedad, y nunca delega la gerencia de este puesto (Musetta, 2009).

El modelo de línea dura y blanda de Guhl es un modelo teórico. En la línea dura, se enfoca en aumentar la oferta de agua y hacerla más estable en el tiempo y en el espacio, mediante la construcción de obras de infraestructura que permiten contar con una oferta mayor y más estable de agua a lo largo del año, o una disponibilidad en zonas que presentan déficit. Su objetivo fundamental es la conservación de la oferta de agua y su uso eficiente. Da consideración integral al ciclo hidrológico con base en cambios de actitudes y comportamientos de los usuarios con respecto a la valoración del agua como un recurso finito y la consecuente limitación de su disponibilidad y el desarrollo y empleo de tecnologías más eficientes (Guhl, 2008).

\section{Resultados y discusión}

\section{Propuesta de adopción de un modelo de gobernanza}

Como se ha referido existen varios modelos aplicados en diferentes lugares del mundo, cada uno con ciertas particularidades. En función de las características propias del sistema acuífero de La Yarada, por la intervención del Estado en las actividades económicas de la región y del país, podemos concluir que el modelo leviatán hidráulico de Mussetta es el mejor. Se adecua a las particularidades del acuífero en cuestión y es un modelo fuerte en términos de presencia estatal, que planifica, desarrolla, construye obras de infraestructura hidráulica. Es a su vez el Estado que organiza a los demás actores de la economía y de la sociedad, y nunca delega la gerencia de este puesto. Este tipo de acciones son las requeridas en un sistema como el estudiado, donde se presenta un desorden generalizado, no se cumplen los reglamentos ni las leyes, y se ha puesto en una situación caótica la estabilidad del sistema acuífero, en tanto que la recarga es muy inferior a la explotación debido a que se han generado usuarios no autorizados a los cuales no se les ha aplicado ninguna sanción.

Un ejemplo claro de debilidad del Estado y sus instituciones se evidencia con la emisión del D.S. N ${ }^{\circ}$ 007-2015-MINAGRI, el cual señala: "Las zonas declaradas en veda mantienen su condición, procediéndose de manera excepcional y por única vez a formalizar o regularizar las licencias de uso de agua, conforme a lo establecido en el presente Decreto Supremo". Este decreto resulta totalmente contradictorio, existiendo la R.M. N ${ }^{\circ}$ 696-98-AG que ratifica la veda en el acuífero de La Yarada. Se decreta la formalización de licencias de pozos no autorizados que vienen generando un gran desbalance en el sistema, lo que ha producido no solo descenso de nivel freático sino también deterioro de la calidad de 
agua por procesos de intrusión marina en gran parte del acuífero, tal como lo han demostrado los estudios realizados por la ANA.

La experiencia post emisión del D.S. $\mathrm{N}^{\circ}$ 007-2015-MINAGRI ha demostrado que la situación se agravó. En el plazo otorgado para la presentación de expedientes se generó una acción masiva y descontrolada de perforaciones de pozos en la zona de estudio, con el fin de lograr la regularización de dicha infraestructura de explotación de aguas subterráneas. Asimismo, posterior al proceso de regularización efectuado, las perforaciones de pozos continúan en forma indiscriminada, con lo que se demuestra que el efecto, más que un paliativo para el problema, resultó un detonante para la perforación de pozos sin control, haciendo caso omiso de la normatividad vigente, y lo más preocupante, se intentó implementar una acción sin los mecanismos de control y seguridad requeridos.

\section{El sistema acuífero de La Yarada}

Según la particularidad del sistema acuífero de La Yarada y la gestión del agua en el Perú, se observa, desde el enfoque de gobernanza del agua, que la presencia y las acciones de grupos sociales han aumentado con los años, ejerciendo una fuerte presión sobre el acuífero. Así, podemos establecer que el tránsito de la gobernabilidad a la gobernanza presenta indicadores de que las instituciones no han manejado adecuadamente sus tareas y responsabilidades y que los agentes sociales han ganado presencia en la desmedida extracción de agua del acuífero, sin que la autoridad pueda intervenir en forma concreta. No se aplican sanciones para infractores de las leyes y dispositivos restrictivos de uso de agua del acuífero, generando un incremento de pozos de extracción que han crecido en forma geométrica en los últimos años, con el consiguiente deterioro de la calidad del agua.

\section{Conclusión}

La política de gobierno genera una grave colisión entre la R.M. $\mathrm{N}^{\circ}$ 696-98-AG que ratifica la veda en el acuífero de La Yarada y el D.S. $\mathrm{N}^{\circ}$
007-2015-MINAGRI, que autoriza para formalizar o regularizar las licencias de uso de agua en el referido acuífero. Esta situación ha generado un antecedente negativo de la política gubernamental en la administración y manejo del agua y en general de todos los recursos naturales sobre el territorio peruano.

En el acuífero de La Yarada, por el problema de gobernabilidad y gobernanza se ven alteradas y rebasadas las leyes respecto a los regímenes de la gestión y limitación de los recursos hídricos subterráneos. Ambos presentan cualidades que podrían brindar un beneficio relevante para el aprovechamiento sostenible de los recursos hídricos, pero de no tomarse acciones inmediatas por parte del aparato gubernamental, la crisis del sistema podría volverse irreversible, con el consiguiente perjuicio económico que esto acarrea. En la zona se cuenta con grandes extensiones de plantaciones de olivo, que son el soporte de la actividad económica en la región.

En el Perú resulta necesario contar con un marco normativo específico para las aguas subterráneas que reconozca su particularidad. Se debe consolidar y hacer fuerte la institucionalidad en materia de Derecho de Aguas y esta no es una necesidad única para el caso de las aguas subterráneas, sino de manera general para los recursos hídricos en todas sus formas. Por tanto, urge la participación eficiente y transparente de la administración pública que permita una gestión adecuada del agua para un aprovechamiento sostenible. Podemos adoptar el modelo leviatán hidráulico de Mussetta, fuerte en términos de presencia estatal, que planifica, desarrolla, construye obras de infraestructura hidráulica. A su vez el Estado es el que organiza a los demás actores de la economía y de la sociedad, y no delega funciones. Este tipo de acciones son las requeridas en un sistema como el estudiado, donde se presenta un desorden generalizado, no se cumplen los reglamentos ni las leyes, y se ha puesto en una situación caótica la estabilidad del sistema acuífero, en tanto que la recarga es muy inferior a la explotación debido a que se han generado usuarios no autorizados a los cuales no se les ha aplicado ninguna sanción. 


\section{Literatura citada}

Borchers, J.; Carpenter, M.; Grabert, V.; Dalgish, B.; Cannon, D. 2015. Land Subsidence from Groundwater Use in California. California Water Foundation. California, USA. 193 p.

Burchi, S.; Mechlem, K.

2007. Agua subterránea en el Derecho internacional; Recopilación de tratados y otros instrumentos legales. FAO Estudio legislativo 86. Roma. Italia. Disponible en: http://www.fao.org/docrep/008/ y5739e/y5739e00.htm\#Contents. Consultado: 10/abr/2018

Cairampoma, A.; Villegas, P.

2016. Régimen jurídico de las aguas subterráneas en el Perú. THËMIS Revista de Derecho, 1 (69): 147-158.

Dourojeanni, A.; Jouravlev, A.

2001. Crisis de Gobernabilidad en la Gestión del Agua (Desafíos que enfrenta la implementación de recomendaciones contenidas en el capítulo 18 del Programa 21). CEPAL. Santiago de Chile. Chile. 83 p.

Fung, A., \& Wright, E.

2003. Deepening Democracy: Innovations in Empowered Participatory Governance. Verso. London-New York. 314 p.

González, N.

2017. Desafíos de la gobernanza ambiental: una aproximación a las implicaciones de la Gestión Integrada del Recurso Hídrico en Colombia. Ciencia Política, 12 (23): 205-229.

Green, S.; Peterson, W.

2005. Governance in Contemporary, The Semisovereign State Revisited. Cambridge University Press. New York. USA. 334 p.

Guhl, E.

2008. Hacia una Gestión Integrada del Agua en la Región Andina. AECID. Bogotá, Colombia. 168 p.

Healey, P.

1997. Collaborative Planning: Shaping Places in Fragmented Societies. UBS Press. Vancouver, Canada. 339 p.

Kersbergen, K.; Waarden, F.

2004. 'Governance' as a bridge between disciplines: Crossdisciplinary inspiration regarding shifts in governance and problems of governability, accountability and legitimacy. European Journal of Political Research, 43: 143-171.

Minaverry, C.

2017. Consumidores y usuarios del servicio del agua en Argentina. Enfoques jurídicos. Tecnología y Ciencias del Agua, 8 (1): 5-20.

Mohamed, E.; Lashin, A.; Abdalla, F.; Al-Bassam, A.

2017. Assessing the hydrogeochemical processes affecting groundwater pollution in arid areas using an integration of geochemical equilibrium and multivariate statistical techniues. Environmental Pollution, 229: 760-770.
Musetta, P.

2009. Participación y gobernanza. El modelo de gobierno del agua en México. Espacios Públicos, 12 (25): 66-84.

Retamal, M.; Andreoli, A.; Arumi, J.; Rojas, J.; Parra, O.

2013. Gobernanza del Agua y Cambio Climático: Fortalezas y Debilidades del Actual Sistema de Gestión del Agua en Chile. Análisis Interno. Interciencia, 38 (1): 8-16.

Rodríguez, M.

2005. La posible creación de mercados de agua y la gobernabilidad de este recurso en Colombia. Comentarios sobre el proyecto de ley del agua. Revista de Ingeniería, 22: 94-102.

Silva, J.

2016. Propuesta de un modelo de gobernanza del agua. XX Congreso Internacional de Contaduría, Administración e Informática de la UNAM. México.

Terence, R.; Jouravlev, A.

1998. Princes, property and markets in water allocaton, (LC/L 1097). Comisión para América Latina y el Caribe (Cepal). Santiago de Chile. 84p.

Todd, F.

2015. New NASA data show how the world is running out of water. The Washington Post. USA. Disponible en: https:// www.independent.co.uk/environment/nasa-data-shows-theworld-is-running-out-of-water-10325188.html. Consultado el $10 / \mathrm{abr} / 18$.

UNESCO.

2006. El agua, una responsabilidad compartida. Segundo informe de las Naciones Unidas sobre el desarrollo de los recursos hídricos en el mundo. UNESCO. 52p.

UNESCO.

2015. Gobernanza de las aguas subterráneas en los acuíferos transfronterizos (Proyecto GGRETA). PANORAMA Y RESULTADOS DE LA ETAPA DE EVALUACIÓN (2013-2015). París, Francia. 15p.

Villalobos, O.

2015. ¿CUÁLES LADIFERENCIAENTREGOBERNABILIDAD Y GOBERNANZA? Journal Rural.com. Disponible en: http://journalrural.com/gobernabilidad-y-gobernanzalocal/\#more-2407. Consultado el 15/4/18.

WWAP.

2006. Water a shared responsibility. The United Nations World Water Development Report 2. UNESCO. France. 584p.

Zamudio, C.

2012. Governance of water resources in Colombia: between progress and challenges. Gestión y Ambiente, 15 (3): 99-112. 
